\title{
MAPPING OF FOLIAR CONTENT USING RADIATIVE TRANSFER MODELING AND VIS-NIR HYPERSPECTRAL CLOSE-RANGE REMOTE-SENSING
}

\author{
S. Jay ${ }^{\text {a* }}$, R. Bendoula ${ }^{a}$, X. Hadoux ${ }^{\mathrm{a}}$, N. Gorretta ${ }^{\mathrm{a}}$ \\ ${ }^{a}$ UMR ITAP, Irstea, 361 rue J.F. Breton, 34196 Montpellier, France \\ sylvain.jay@irstea.fr
}

KEY WORDS: Close-range remote sensing, Hyperspectral, Leaf optical properties, Radiative transfer model, Vegetation

\begin{abstract}
:
Most methods for retrieving foliar content from hyperspectral data are well adapted either to remote-sensing scale, for which each spectral measurement has a spatial resolution ranging from a few dozen centimeters to a few hundred meters, or to leaf scale, for which an integrating sphere is required to collect the spectral data. In this study, we present a method for estimating leaf optical properties from hyperspectral images having a spatial resolution of a few millimeters or centimeters. In presence of a single light source assumed to be directional, it is shown that leaf hyperspectral measurements can be related to the directional hemispherical reflectance simulated by the PROSPECT radiative transfer model using two other parameters. The first one is a multiplicative term that is related to local leaf angle and illumination zenith angle. The second parameter is an additive specular-related term that models BRDF effects.

Our model was tested on visible and near infrared hyperspectral images of leaves of various species, that were acquired under laboratory conditions. Introducing these two additional parameters into the inversion scheme leads to improved estimation results of PROSPECT parameters when compared to original PROSPECT. In particular, the RMSE for local chlorophyll content estimation was reduced by $21 \%$ (resp. 32\%) when tested on leaves placed in horizontal (resp. sloping) position. Furthermore, inverting this model provides interesting information on local leaf angle, which is a crucial parameter in classical remote-sensing.
\end{abstract}

\section{INTRODUCTION}

For the last decades, hyperspectral remote-sensing has proven to offer a great potential for extracting vegetation optical properties at different scales, ranging from leaf level to canopy level. Such a non-destructive technique allows the assessment of spatial and temporal variability of vegetation status, which is useful for many environmental, ecological and agricultural applications such as early detection of leaf diseases (Mahlein et al., 2013).

Because these optical properties affect the reflected and transmitted incoming radiations, they can be retrieved from the spectral shape of the measured signal under some conditions. In the solar emission spectrum (i.e., between 400 and $2500 \mathrm{~nm}$ ), various parameters are known to have an influence in some specific wavelength ranges. At leaf level, these parameters characterize the leaf internal structure and biochemical composition through absorption and scattering processes, e.g., chlorophyll, water and dry matter contents (Curran, 1989; Jacquemoud and Baret, 1990; Feret et al., 2008). They also describe leaf surface properties (Bousquet et al., 2005). At canopy level, they characterize the canopy structure, e.g., leaf area index or leaf inclination distribution function (Knyazikhin et al., 2012; Latorre-Carmona et al., 2014; Verhoef, 1984), thus resulting in complex relationships with re-emitted light. In addition, depending on the study scale, other factors have to considered, such as soil properties and atmospheric conditions (Jacquemoud et al., 2009).

All these parameters determine the bi-directional reflectance distribution function (BRDF) that describes the angular patterns of reflected light, as leaf-surface characteristics can make vegetation highly non-Lambertian (Comar et al., 2012). Because hyperspectral measurements are affected by BRDF effets, it is of great importance to take into account all these sources of variability to relate remote-sensing data to leaf physiological processes in a proper way.

\footnotetext{
${ }^{*}$ Corresponding author
}

A number of retrieval methods have been developed, each of which has advantages and drawbacks as reviewed by Baret and Buis (2008) and Dorigo et al. (2007). They can roughly be classified into two main classes, i.e., statistically- and physically-based methods. Statistical methods aim at finding a statistical relationship between the spectral signature and targeted variable(s) (Gitelson et al., 2005; Jay et al., 2014). Conversely, physically-based methods lie in the use of radiative transfer models simulating light propagation within the leaf and/or canopy, and therefore, they do not require any training data base. At leaf level, these models range from simple plate models to ray-tracing, radiosity and stochastic models that are computationally more difficult to invert directly (Dorigo et al., 2007). PROSPECT (Jacquemoud and Baret, 1990) is one the most known plate models, probably because of its suitability to retrieve leaf biochemical contents by model inversion, its ease of use and its availability. At canopy level, various radiative transfer models have also been developed since SAIL, one of the earliest and most known models (Verhoef, 1984). Inversion of canopy models allows the estimation of parameters related to canopy structure, such as leaf area index or leaf inclination distribution function. The combination of both leaf-level and canopy-level models, e.g. PROSAIL (Jacquemoud et al., 2009), therefore allows the estimation of leaf biochemical parameters from remote-sensing observations.

However, these radiative transfer models are well adapted to specific resolution ranges and measurement setups. At leaf level, because PROSPECT models directional hemispherical reflectance and transmittance, its inversion requires the use of integrating spheres to collect the spectral data (Jacquemoud and Baret, 1990; Feret et al., 2008). On the other hand, canopy reflectance models such as PROSAIL must be applied to mixed pixels (containing both soil and leaf materials), for which effects of leaf composition, canopy structure, soil properties and viewing/illumination angles are integrated into a single spectrum. They are well suited for airborne and satellite-borne hyperspectral measurements as well as ground-based spectroradiometric measurements, for which 
the spatial resolution is generally coarser than a few dozen centimeters (Zarco-Tejada et al., 2003; Schlemmer et al., 2013).

However, they cannot be applied to hyperspectral data having a higher spatial resolution (down to centimeter or millimeter level) because the assumption of mixed pixel does not hold any more. Such close-range remote-sensing hyperspectral data allow more detailed spatial and spectral descriptions of vegetation status, and may become increasingly available with recent developments of unmanned aerial vehicles (Duan et al., 2014; Verger et al., 2014). Only few studies have dealt with such images (Vigneau et al., 2011; Uto and Kosugi, 2013), perhaps because of the heterogeneity of illumination conditions that makes difficult the reflectance correction process in every pixel. Indeed, the reference surface used for reflectance correction is not submitted to the same illumination conditions than vegetation pixels, e.g., because of shadows or multiple scattering caused by nearby leaves.

Furthermore, the reflectance variations with respect to illumination and viewing angles (i.e., BRDF effets) have to be carefully addressed, as they can greatly affect the measured radiance (Bousquet et al., 2005; Comar et al., 2012).

In this study, we address the estimation of both the foliar content and leaf topography from such high-resolution hyperspectral data in the visible and near-infrared range. We present a physicallybased radiance model that relates both leaf biochemistry and local incident angle to the acquired signal in presence of a single light source (e.g., under laboratory conditions). This model is derived from the PROSPECT model and its inversion allows the simultaneous retrieval of PROSPECT parameters (e.g., chlorophyll and carotenoid contents), leaf surface effects and leaf angle with respect to the light source.

In this article, the whole theory is described in Section 2.. Experiments and results are presented in Section 3., and we finally draw some conclusions in Section $4 .$.

\section{THEORY}

\subsection{Radiometric definitions}

Before going into further details, it is highly necessary to define the main physical quantities that will be used in the following article. These definitions and notations are based on the initial terminology of Nicodemus et al. (1977) and have been recalled by Schaepman-Strub et al. (2006).

The spectral radiance $L$ is the radiant flux in a beam per unit wavelength, per unit area and per unit solid angle, and is expressed in the SI unit $\left[\mathrm{W} \cdot \mathrm{sr}^{-1} \cdot \mathrm{m}^{-2} \cdot \mathrm{nm}^{-1}\right]$. This is the physical quantity measured by a hyperspectral imaging sensor after spectral calibration. Similarly, the spectral irradiance $E$ is the radiant flux in a beam per unit wavelength and per unit area and is expressed in $\left[\mathrm{W} \cdot \mathrm{m}^{-2} . \mathrm{nm}^{-1}\right]$.

One of the main physical quantities used to describe angular patterns of reflected light is the spectral bidirectional reflectance distribution function (BRDF) expressed in $\left[\mathrm{sr}^{-1}\right]$. It describes how a parallel beam of incident light from one direction in the hemisphere is reflected into another direction in the hemisphere:

$$
f_{r}\left(\lambda, \theta_{s} ; \theta_{v}, \varphi_{v}\right)=\frac{d L_{r}\left(\lambda, \theta_{s} ; \theta_{v}, \varphi_{v}\right)}{d E_{i}\left(\lambda, \theta_{s}\right)} .
$$

Because it is the ratio of two infinitesimal quantities, it cannot theoretically be measured. However, its integration over the corresponding solid angles allows the derivation of many other measurable physical quantities.

Usually, the reflectance correction process does not consist in retrieving directly the reflectance (defined as the ratio of the leaving radiant exitance to the incident irradiance), but rather follows the definition of a reflectance factor. In the specific case of single illumination and viewing directions, the bidirectional reflectance factor (BRF) is given by the ratio of the radiant flux $d L_{r}$ reflected from the area element dA to the radiant flux $d L_{r}^{i d}$ reflected from an ideal and diffuse surface of the same area dA under identical illumination and viewing geometries. It is unitless and as developed by Schaepman-Strub et al. (2006), it is given by:

$$
R\left(\lambda, \theta_{s} ; \theta_{v}, \varphi_{v}\right)=\frac{d L_{r}\left(\lambda, \theta_{s} ; \theta_{v}, \varphi_{v}\right)}{d L_{r}^{i d}\left(\lambda, \theta_{s}\right)}
$$

where $d L_{r}^{i d}$ does not depend on viewing angles.

Because the BRDF of an ideal and diffuse surface is $(1 / \pi)$, the $\mathrm{BRF}$ of any surface is therefore given by:

$$
R\left(\lambda, \theta_{s} ; \theta_{v}, \varphi_{v}\right)=\pi f_{r}\left(\lambda, \theta_{s} ; \theta_{v}, \varphi_{v}\right) .
$$

Lastly, is it worth defining the directional-hemispherical reflectance (DHR) as the integration of BRDF over the whole viewing hemisphere. The DHR is unitless and is given by:

$\rho\left(\lambda, \theta_{s} ; 2 \pi\right)=\int_{0}^{2 \pi} \int_{0}^{\pi / 2} f_{r}\left(\lambda, \theta_{s} ; \theta_{v}, \varphi_{v}\right) \cos \theta_{v} \sin \theta_{v} d \theta_{v} d \varphi_{v}$.

In the following, the spectral dependence will be omitted for more clarity in notation. Similarly, spectral radiance and spectral irradiance will be simply referred to as radiance and irradiance.

\subsection{Relations with hyperspectral measurement}

Generally, sensor measurements allow the retrieval of a biconical reflectance factor because the solid angles corresponding to incident and reflected light are never purely directional (SchaepmanStrub et al., 2006). The cone sizes can vary from nearly zero for directional light source and/or small sensor FOV, to $2 \pi$ for hemispherical illumination (e.g. under outdoor conditions) and/or viewing.

However, in the following, we assume that, under laboratory conditions, hyperspectral imaging sensors can allow the retrieval of BRF measurements. To better meet this assumption, one should use a collimated beam to illuminate the imaged line (see Section 3.1), and the pixel FOV has to be very small (which is usually the case for most hyperspectral cameras).

The leaf BRF $R$ is thus approximated as follows:

$$
R\left(\theta_{s} ; \theta_{v}, \varphi_{v}\right) \approx \frac{L_{r}\left(\theta_{s} ; \theta_{v}, \varphi_{v}\right)}{L_{r}^{i d}\left(\theta_{s}\right)}
$$

where $L_{r}$ and $L_{r}^{i d}$ are the radiances measured on the leaf and reference surface respectively.

Importantly, Eq. 5 requires the leaf and reference surface to be under identical illumination and viewing geometries (i.e., same $\theta_{s}$ values). But, at the pixel level, variable leaf position and leaf surface topography can make the irradiances received by the leaf and reference surface highly different. Indeed, in presence of a directional light source, the irradiance received by an area element is proportional to the cosine of the incident angle, i.e., the angle $\theta_{i}$ between the light source and the normal to this element, i.e.:

$$
E_{i}\left(\theta_{i}\right)=E_{0} \cos \left(\theta_{i}\right)
$$

where $E_{0}$ is the irradiance received by an area element perpendicular to the light source axis, and $\cos \theta_{i}=\cos \theta_{s} \cos \theta_{l}+\sin \theta_{s} \sin \theta_{l} \cos \varphi_{l}$ (Bousquet et al., 2005; Comar et al., 2014).

Therefore, we define two illumination geometries, each of which corresponds to either the leaf or reference surface. The irradiance 
received by the leaf is:

$$
E_{i}^{l}\left(\theta_{i}^{l}\right)=E_{0} \cos \left(\theta_{i}^{l}\right)
$$

whereas the one received by the reference surface is:

$$
E_{i}^{i d}\left(\theta_{i}^{i d}\right)=E_{0} \cos \left(\theta_{i}^{i d}\right) .
$$

Usually, the reference surface is horizontal so, in the following, we simply note $\theta_{i}^{i d}=\theta_{s}$ and $\theta_{i}^{l}=\theta_{i}$.

Similarly to Eq. 5, in each pixel, the physical quantity $R_{\text {hyp }}$ retrieved using a hyperspectral camera is given by:

$$
R_{h y p}\left(\theta_{s}, \theta_{i} ; \theta_{v}, \varphi_{v}\right) \approx \frac{L_{r}\left(\theta_{i} ; \theta_{v}, \varphi_{v}\right)}{L_{r}^{i d}\left(\theta_{s}\right)} .
$$

Assuming the light source is directional and the pixel FOV is small, combining Eq. 1 and Eq. 9 leads to the following equation:

$$
R_{h y p}\left(\theta_{s}, \theta_{i} ; \theta_{v}, \varphi_{v}\right) \approx \frac{f_{r}\left(\theta_{i} ; \theta_{v}, \varphi_{v}\right) E_{i}^{l}\left(\theta_{i}\right)}{(1 / \pi) E_{i}^{i d}\left(\theta_{s}\right)}
$$

Using Eq. 7 and Eq. 8, $R_{\text {hyp }}$ can finally be expressed as follows:

$$
R_{\text {hyp }}\left(\theta_{s}, \theta_{i} ; \theta_{v}, \varphi_{v}\right) \approx R\left(\theta_{i} ; \theta_{v}, \varphi_{v}\right) \frac{\cos \theta_{i}}{\cos \theta_{s}}
$$

Eq. 11 reveals that the physical reflectance quantity retrieved from a small FOV sensor in presence of a directional light source is not directly the leaf BRF if the leaf and reference surface are differently tilted toward the light source. We denote it as a leaf pseudo $\mathrm{BRF}$, i.e., the leaf BRF weighted by the ratio of the cosine of the angle between the light source and the normal to the leaf, to the cosine of illumination zenith angle.

Therefore, Eq. 11 relates the measured pseudo BRF to the (local) incident angle and leaf BRF. To relate the latter to the foliar content, it is then necessary to take into account leaf surface properties as described in the next section.

\subsection{A PROSPECT-based leaf radiance model}

At leaf level, the PROSPECT model has been widely used to model the relationship between the DHR of various monocotyledon and dicotyledon species and their biochemical content and leaf structure. The original version developed by Jacquemoud and Baret (1990) has been successively improved over the years to take into account other biochemical components and larger spectral ranges (Jacquemoud et al., 1996; Baret and Fourty, 1997; Jacquemoud et al., 2000; Feret et al., 2008; Gerber et al., 2011). Because it simulates the sum of both specular and diffuse reflected fluxes over the whole hemisphere, its inversion requires the use of integrating spheres to collect the reflected flux. As a result, it cannot directly be applied to hyperspectral remotesensing observations of vegetation that are affected by BRDF effects. An important prerequisite before using PROSPECT in a remote-sensing context, is therefore to relate the leaf DHR to the leaf BRDF.

First, it is generally admitted that the leaf BRDF is the sum of a diffuse component $f_{r, \operatorname{diff}}$ and a directional component $f_{r, s p e c}$ (Comar et al., 2014; Bousquet et al., 2005):

$$
f_{r}\left(\theta_{i} ; \theta_{v}, \varphi_{v}\right)=f_{r, \text { spec }}\left(\theta_{i} ; \theta_{v}, \varphi_{v}\right)+f_{r, d i f f}\left(\theta_{i} ; \theta_{v}, \varphi_{v}\right) .
$$

The diffuse component $f_{r, \text { diff }}$ characterizes absorption and scattering processes within the leaf volume and thus depends on opticallyactive biochemical compounds. The directional component $f_{r, s p e c}$ characterizes leaf surface properties and describes how light is reflected at the surface. In the visible and near-infrared range, $f_{r, \text { spec }}$ is assumed to be wavelength-independent, whereas $f_{r, \text { dif } f}$ highly varies with the wavelength (Comar et al., 2014; Bousquet et al., 2005).

Bousquet et al. (2005) have developed a leaf BRDF model for both $f_{r, s p e c}$ and $f_{r, d i f f}$. The modeled directional component depends on illumination and viewing geometries as well as on the wax refraction index and a roughness parameter. The modeled diffuse component is given by the Lambert coefficient $k_{l}$ divided by $\pi$.

However, a more accurate way to take into account the two-layer leaf structure (i.e. composed of an upper wax layer and a bottom leaf mesophyll layer) is to consider the fraction of light that is reflected by the first layer and that does not reach the bottom layer (Stuckens et al., 2009; Ashikmin et al., 2000). The diffuse component is then expressed as a function of leaf mesophyll Lambert coefficient $k_{l}$ and wax DHR $\rho_{\text {spec }}$ as follows (Stuckens et al., 2009):

$$
f_{r, \text { diff }}\left(\theta_{i} ; \theta_{v}, \varphi_{v}\right)=\frac{k_{l}}{\pi}\left(1-\rho_{\text {spec }}\left(\theta_{i} ; 2 \pi\right)\right) .
$$

Combining Eq. 4, Eq. 12 and Eq. 13 leads to the following expression for total DHR :

$$
\begin{aligned}
& \rho\left(\theta_{i} ; 2 \pi\right)=\int_{0}^{2 \pi} \int_{0}^{\pi / 2} f_{r, s p e c}\left(\theta_{i} ; \theta_{v}, \varphi_{v}\right) \cos \theta_{v} \sin \theta_{v} d \theta_{v} d \varphi_{v} \\
& +\frac{k_{l}}{\pi}\left(1-\rho_{\text {spec }}\left(\theta_{i} ; 2 \pi\right)\right) \int_{0}^{2 \pi} \int_{0}^{\pi / 2} \cos \theta_{v} \sin \theta_{v} d \theta_{v} d \varphi_{v} .
\end{aligned}
$$

This equation can be rewritten as follows:

$$
\rho\left(\theta_{i} ; 2 \pi\right)=\rho_{\text {spec }}\left(\theta_{i} ; 2 \pi\right)+k_{l}\left(1-\rho_{\text {spec }}\left(\theta_{i} ; 2 \pi\right)\right) .
$$

Because PROSPECT models the relationship between leaf DHR for nadir illumination $\rho(0 ; 2 \pi)$ and leaf biochemical content characterized by the vector of PROSPECT input parameters $\boldsymbol{\vartheta}_{\text {prospect }}$, the modeled Lambert coefficient can be expressed as a function of $\rho_{\text {spec }}(0 ; 2 \pi)$ and PROSPECT DHR $\rho_{\text {prospect }}$ using Eq. 15 as follows:

$$
k_{l, \text { mod }}\left(\boldsymbol{\vartheta}_{\text {prospect }}\right)=\frac{\rho_{\text {prospect }}\left(\boldsymbol{\vartheta}_{\text {prospect }}\right)-\rho_{\text {spec }}(0 ; 2 \pi)}{1-\rho_{\text {spec }}(0 ; 2 \pi)} .
$$

Subsequently, the leaf BRDF can be modeled as a function of PROSPECT parameters using Eq. 12, Eq. 13 and Eq. 16:

$$
\begin{aligned}
& f_{r, \text { mod }}\left(\boldsymbol{\vartheta}_{\text {prospect }}, \theta_{i} ; \theta_{v}, \varphi_{v}\right)=f_{r, \text { spec }}\left(\theta_{i} ; \theta_{v}, \varphi_{v}\right) \\
+ & \frac{1}{\pi}\left(\frac{1-\rho_{\text {spec }}\left(\theta_{i} ; 2 \pi\right)}{1-\rho_{\text {spec }}(0 ; 2 \pi)}\right)\left(\rho_{\text {prospect }}\left(\boldsymbol{\vartheta}_{\text {prospect }}\right)-\rho_{\text {spec }}(0 ; 2 \pi)\right)
\end{aligned}
$$

In order to estimate $\boldsymbol{\vartheta}_{\text {prospect }}$ from the pseudo BRF $R_{\text {hyp }}$ measured by a hyperspectral camera, Eq. 2, Eq. 11 and Eq. 17 are combined to lead to the following leaf pseudo BRF model:

$$
\begin{aligned}
R_{\text {hyp }, \text { mod }}\left(\boldsymbol{\vartheta}_{\text {prospect }}, \theta_{s}, \theta_{i} ; \theta_{v}, \varphi_{v}\right)= \\
\left(\frac{\cos \theta_{i}}{\cos \theta_{s}}\right)\left(\frac{1-\rho_{\text {spec }}\left(\theta_{i} ; 2 \pi\right)}{1-\rho_{\text {spec }}(0 ; 2 \pi)}\right) \rho_{\text {prospect }}\left(\boldsymbol{\vartheta}_{\text {prospect }}\right) \\
+\left(\frac{\cos \theta_{i}}{\cos \theta_{s}}\right)\left[\pi f_{r, \text { spec }}\left(\theta_{i} ; \theta_{v}, \varphi_{v}\right)\right. \\
\left.\quad-\rho_{\text {spec }}(0 ; 2 \pi)\left(\frac{1-\rho_{\text {spec }}\left(\theta_{i} ; 2 \pi\right)}{1-\rho_{\text {spec }}(0 ; 2 \pi)}\right)\right]
\end{aligned}
$$

that has been splitted into a wavelength-dependent term and wavelengthindependent term. In this expression, $f_{r, \text { spec }}$ and $\rho_{\text {spec }}$ could potentially be replaced using the BRDF model developed by Bous- 
quet et al. (2005), thus being parameterized as a function of $\theta_{i}$, $\theta_{v}, \varphi_{v}$, the wax refraction index $n$ and roughness parameter $\sigma$. Unfortunately, such a model is over-parameterized so its inversion is a degenerate problem and leads to poor estimation results (not shown here). However, because the second term does not depend on wavelength, Eq. 18 can be simplified as follows:

$$
\begin{aligned}
R_{\text {hyp }, \text { mod }}\left(\boldsymbol{\vartheta}_{\text {prospect }}, \theta_{s}, \theta_{i}, b_{\text {spec }}\right) & = \\
\left(\frac{\cos \theta_{i}}{\cos \theta_{s}}\right)\left(\frac{1-\rho_{\text {spec }}\left(\theta_{i} ; 2 \pi\right)}{1-\rho_{\text {spec }}(0 ; 2 \pi)}\right) & \rho_{\text {prospect }}\left(\boldsymbol{\vartheta}_{\text {prospect }}\right) \\
& +\left(\frac{\cos \theta_{i}}{\cos \theta_{s}}\right) b_{\text {spec }}
\end{aligned}
$$

where $b_{\text {spec }}=b_{\text {spec }}\left(\theta_{i} ; \theta_{v}, \varphi_{v}\right)=\pi f_{r, \text { spec }}\left(\theta_{i} ; \theta_{v}, \varphi_{v}\right)-$ $\rho_{\text {spec }}(0 ; 2 \pi)\left(\frac{1-\rho_{\text {spec }}\left(\theta_{i} ; 2 \pi\right)}{1-\rho_{\text {spec }}(0 ; 2 \pi)}\right)$. Furthermore, if we assume that, for low $\theta_{i}$ values, the fraction $\frac{1-\rho_{\text {spec }}\left(\theta_{i} ; 2 \pi\right)}{1-\rho_{\text {spec }}(0 ; 2 \pi)}$ is nearly one, Eq. 19 can finally be approximated by the following leaf pseudo BRF model:

$$
\begin{aligned}
& R_{\text {hyp }, \text { mod }}\left(\boldsymbol{\vartheta}_{\text {prospect }}, \theta_{s}, \theta_{i}, b_{\text {spec }}\right)= \\
& \quad\left(\frac{\cos \theta_{i}}{\cos \theta_{s}}\right) \rho_{\text {prospect }}\left(\boldsymbol{\vartheta}_{\text {prospect }}\right)+\left(\frac{\cos \theta_{i}}{\cos \theta_{s}}\right) b_{\text {spec }} .
\end{aligned}
$$

This model relates the pseudo BRF to the incident angle $\theta_{i}$, foliar content through the PROSPECT parameters $\boldsymbol{\vartheta}_{\text {prospect }}$ and leaf surface effect $b_{\text {spec }}$. Importantly, this model is only well suited for low incident angles so further investigation will be necessary for higher angles.

Lastly, because the hyperspectral camera acquires radiance values, Eq. 9 is used to transform the above pseudo BRF model into a radiance model as follows:

$$
\begin{aligned}
L_{r, \text { mod }}\left(\boldsymbol{\vartheta}_{\text {prospect }}, \theta_{s}, \theta_{i} ; \theta_{v}, \varphi_{v}\right)= \\
\quad\left[\left(\frac{\cos \theta_{i}}{\cos \theta_{s}}\right) \rho_{\text {prospect }}\left(\boldsymbol{\vartheta}_{\text {prospect }}\right)\right. \\
\left.\quad+\left(\frac{\cos \theta_{i}}{\cos \theta_{s}}\right) b_{\text {spec }}\left(\theta_{i} ; \theta_{v}, \varphi_{v}\right)\right] L_{r}^{i d}\left(\theta_{s}\right) .
\end{aligned}
$$

where $L_{r}^{i d}\left(\theta_{s}\right)$ is estimated on the reference surface. Performing the inversion directly on acquired radiance values instead of retrieved pseudo BRF values is more stable and accurate.

\section{EXPERIMENTS AND RESULTS}

\subsection{Data acquisition}

To test our methods, close-range remote-sensing hyperspectral images have been collected in laboratory conditions. A Hyspex push-broom hyperspectral camera (Norsk Elektro Optikk, Norway) was facing towards nadir at thirty centimeters above the leaves. It acquired successive lines of 1600 pixels and 160 spectral bands ranging from 410 to $1000 \mathrm{~nm}$ with a $3.7 \mathrm{~nm}$ spectral sampling interval. The pixel FOV was 0.18 and $0.36 \mathrm{mrad}$ across and along track respectively. The lighting was provided by a collimated halogen source placed close to the camera with an illumination angle $\theta_{s}=20^{\circ}$. This source was illuminating the same line than that imaged by the camera. The incoming halogen irradiance was estimated by using a reference surface (Spectralon, Labsphere) horizontally placed next to the imaged leaf. After acquisition, each image was calibrated to spectral radiance.

Leaves from six species (bamboo, bramble, ivy, Viburnum tinus, laurel and holly) were imaged in three positions corresponding to (a)

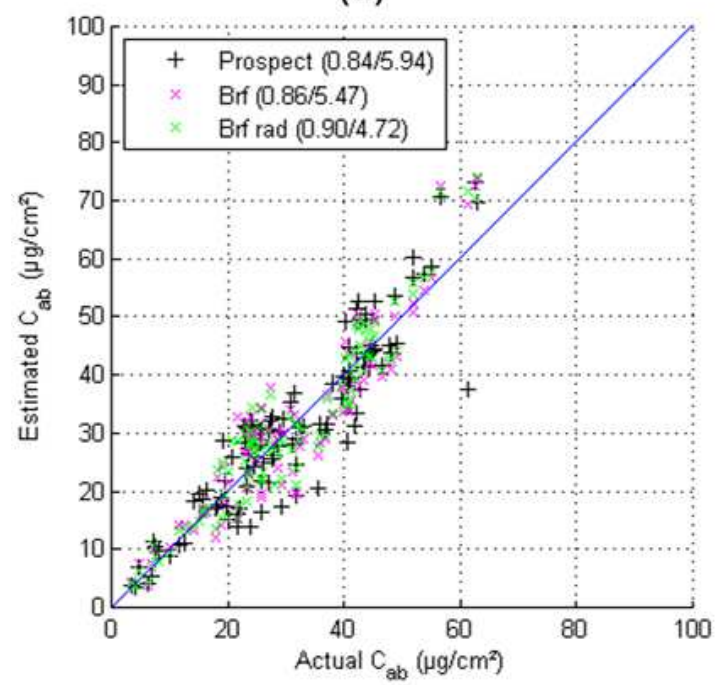

(b)

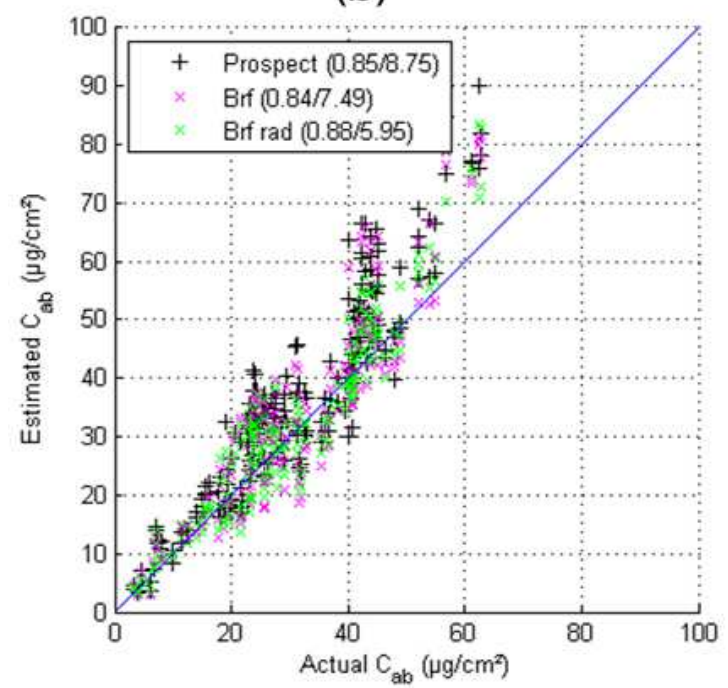

Figure 1: $C_{a b}$ estimation results: (a) flat leaves, and (b) tilted leaves. For each method, the $\mathrm{R}^{2}$ and RMSE are indicated in brackets.

average incident angles $\theta_{i}=0^{\circ}, \theta_{i}=20^{\circ}$ and $\theta_{i}=40^{\circ}$. Subsequently, for each leaf, several leaf discs (from two to seven discs depending on leaf size) were sampled using a cork borer and a Dualex (Force-A, Orsay, France) was used to measure $C_{a b}$ in every disc. The leaf was then photographed to record disc positions.

\subsection{Inversion implementation}

In this article, we used the version $5 b$ of PROSPECT originally developed by Feret et al. (2008). This model takes into account the leaf structure parameter $N$, the chlorophyll a+b content $C_{a b}$, the total carotenoid content $C_{c x}$, the brown pigment content $C_{b p}$, the equivalent water thickness $C_{w}$ and the dry matter content $C_{m}$. Because water absorption is weak between 410 and $1000 \mathrm{~nm}$ and only occurs around $970 \mathrm{~nm}$ (Curran, 1989), the spectral range was restricted to $410-900 \mathrm{~nm}$ and $C_{w}$ was fixed to $C_{w}=0.01 \mathrm{~m}^{-1}$ so as to lower the estimation uncertainty. Therefore, the vector of parameters to estimate $\boldsymbol{\vartheta}$ is given by $\boldsymbol{\vartheta}=\left[N, C_{a b}, C_{c x}, C_{b p}, C_{m}, b_{s p e c}, \theta_{i}\right]^{t}$. The models of Eq. 20 and Eq. 21 were inverted in each pixel using least square optimization implemented in Matlab. To do so, 
(a)

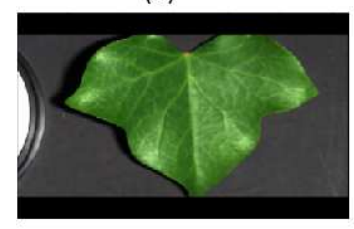

(e)

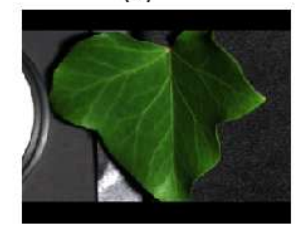

(b)

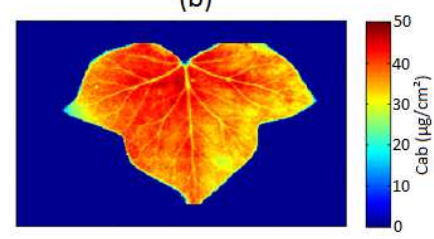

(f)

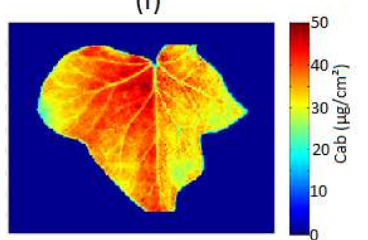

(c)

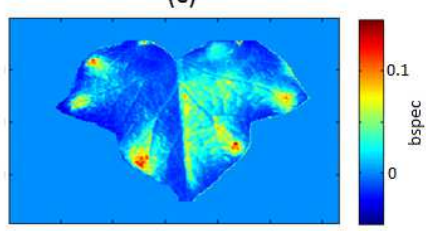

(g)

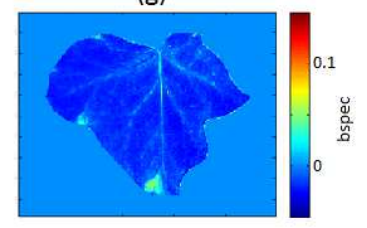

(d)

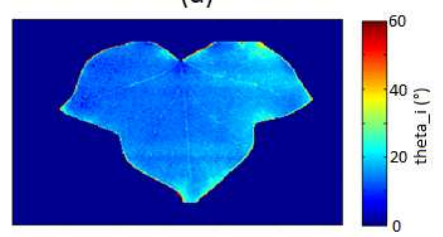

(h)

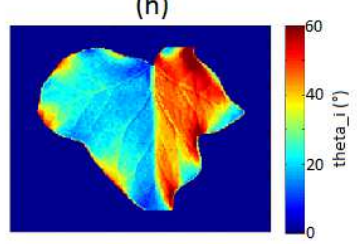

Figure 2: Estimated maps obtained with the radiance model and on the same leaf in flat (a-d) and $40^{\circ}$ tilted (e-h) positions: (a) and (e) RGB images, (b) and (f) $C_{a b}$ estimated maps, (c) and (g) $b_{\text {spec }}$ estimated maps, (d) and (h) $\theta_{i}$ estimated maps.

the following cost function was used (for Eq. 21):

$$
\widehat{\vartheta}=\underset{\vartheta}{\operatorname{argmin}} \sum_{\lambda_{i}}\left[L_{r}\left(\lambda_{i}\right)-L_{r, \bmod }\left(\lambda_{i}, \boldsymbol{\vartheta}\right)\right]^{2}
$$

where $L_{r}\left(\lambda_{i}\right)$ is the measured spectral radiance.

\subsection{Estimation results}

In Fig. 1, we compare the $C_{a b}$ estimation results obtained by original PROSPECT, the pseudo-BRF model of Eq. 20 (noted BRF) and the radiance model of Eq. 21 (noted BRF RAD). Only leaves in flat (resp. tilted) position are used in Fig. 1a (resp. Fig. 1b).

For flat leaves, PROSPECT led to a lower estimation accuracy $\left(\mathrm{R}^{2}=0.84, \mathrm{RMSE}=5.94 \mu \mathrm{g} . \mathrm{cm}^{-2}\right)$ than the pseudo-BRF model $\left(\mathrm{R}^{2}=0.86\right.$, RMSE $\left.=5.47 \mu \mathrm{g} . \mathrm{cm}^{-2}\right)$ and the radiance model $\left(\mathrm{R}^{2}=0.90, \mathrm{RMSE}=4.72 \mu \mathrm{g} . \mathrm{cm}^{-2}\right.$. Similarly, for tilted leaves, the radiance model gave the better results with a $R^{2}$ of 0.88 and a RMSE of $5.95 \mu \mathrm{g} . \mathrm{cm}^{-2}$ ).

The improvement observed for flat leaves is mainly related to the modeling of the specular term. Interestingly, this is well illustrated by the point corresponding to an actual $\mathrm{C}_{a b}$ value of $60 \mu \mathrm{g} . \mathrm{cm}^{-2}$ : while both the radiance and pseudo-BRF models led to reasonable $\mathrm{C}_{a b}$ estimates, PROSPECT inversion largely underestimated $\mathrm{C}_{a b}$. This was due to a local BRDF effect that had caused an overestimation of reflectance beforehand. For tilted leaves, the improvement was even greater (the RMSE was reduced by $32 \%$ for tilted leaves, while it was reduced by $21 \%$ for flat leaves) because of the effect of variable leaf angles.

Lastly, the interest of using directly spectral radiance measurements instead of transformed pseudo-BRF values is clearly demonstrated through these two figures since the RMSE was reduced by at least $14 \%$.

Based on $\mathrm{C}_{a b}$ estimation, Fig. 1 shows that BRDF effects and variable leaf angles are properly taken into account within the proposed radiance model. To illustrate how the specular-related parameter and incident angle are retrieved, in Fig. 2, we display the estimated maps of $C_{a b}, b_{\text {spec }}$ and $\theta_{i}$ for two different leaf positions (flat and tilted with an average incident angle of $40^{\circ}$ ). First, Fig. $2 \mathrm{~b}$ and Fig. $2 \mathrm{f}$ show similar $C_{a b}$ spatial distributions with values ranging from 30 to $45 \mu \mathrm{g} . \mathrm{cm}^{-2}$, i.e., $C_{a b}$ was higher in the left-hand side than in the right-hand side (except in the left leaf tip). As observed in Fig. 2a, there were many BRDF effects within the flat leaf, where illumination and viewing angles relative to the leaf normal were similar. These surface effects were well retrieved by the $b_{\text {spec }}$ parameter as seen in Fig. 2c. Such effects were increasing the reflectance up to 0.14 in this case, thus showing the importance of taking this phenomenon into account, especially for glossy non-Lambertian leaves such as ivy ones. Lastly, the retrieval of incident angle that gives some information on leaf topography is illustrated in Fig. 2d and Fig. 2h. For the flat leaf, the estimated incident angle was similar in the whole leaf, with an average value of $15^{\circ}$ that is close to the illumination zenith angle $\left(\theta_{s}=20^{\circ}\right)$. In Fig. 2d, the highly tilted right-hand side of the leaf observed in Fig. 2e was well retrieved, with $\theta_{i}$ values ranging from 35 to $60^{\circ}$. However, further investigation is needed in case of high incident angles because, as mentioned in Section 2.3, the proposed radiance model was only well suited for low incident angles. In these cases, one should take into account the difference in fluxes reaching the upper wax layer and bottom mesophyll layer.

\section{CONCLUSIONS}

In this study, the PROSPECT model for leaf directional hemispherical reflectance has been adapted to the close-range remotesensing case by adding two parameters describing the specular component and incident angle, which is related to leaf angle and illumination zenith angle. Therefore, inverting this leaf pseudoBRF model allows the estimation of PROSPECT input parameters such as chlorophyll content, as well as leaf local topography. Compared to classical PROSPECT inversion, inverting this model does not require spectral measurements acquired using an integrating sphere. Moreover, model inversion applied on spatially-resolved information provided by hyperspectral imaging allows the high-resolution mapping of foliar content, which can be very useful for early detection of leaf diseases.

\section{ACKNOWLEDGEMENTS}

This study was funded by the French National Research Agency, within the program Investissements davenir with the reference ANR-11-BTBR-0007 (AKER project).

\section{References}

Ashikmin, M., Premože, S. and Shirley, P., 2000. A microfacetbased brdf generator. In: Proceedings of the 27th annual conference on Computer graphics and interactive techniques, ACM Press/Addison-Wesley Publishing Co., pp. 65-74.

Baret, F. and Buis, S., 2008. Estimating canopy characteristics from remote sensing observations: Review of methods and associated problems. In: S. Liang (ed.), Advances in Land Remote Sensing, Springer Netherlands, pp. 173-201. 
Baret, F. and Fourty, T., 1997. Estimation of leaf water content and specific leaf weight from reflectance and transmittance measurements. Agronomie 17(9-10), pp. 455-464.

Bousquet, L., Lachérade, S., Jacquemoud, S. and Moya, I., 2005. Leaf BRDF measurements and model for specular and diffuse components differentiation. Remote Sensing of Environment 98(2), pp. 201-211.

Comar, A., Baret, F., Obein, G., Simonot, L., Meneveaux, D., Vinot, F. and de Solan, B., 2014. ACT: A leaf BRDF model taking into account the azimuthal anisotropy of monocotyledonous leaf surface. Remote Sensing of Environment 143(0), pp. $112-121$.

Comar, A., Baret, F., Vinot, F., Yan, L. and de Solan, B., 2012. Wheat leaf bidirectional reflectance measurements: Description and quantification of the volume, specular and hot-spot scattering features. Remote Sensing of Environment 121(0), pp. $26-35$.

Curran, P. J., 1989. Remote sensing of foliar chemistry. Remote Sensing of Environment 30(3), pp. 271 - 278.

Dorigo, W., Zurita-Milla, R., de Wit, A., Brazile, J., Singh, R. and Schaepman, M., 2007. A review on reflective remote sensing and data assimilation techniques for enhanced agroecosystem modeling. International Journal of Applied Earth Observation and Geoinformation 9(2), pp. $165-193$.

Duan, S.-B., Li, Z.-L., Wu, H., Tang, B.-H., Ma, L., Zhao, E. and $\mathrm{Li}, \mathrm{C} ., 2014$. Inversion of the PROSAIL model to estimate leaf area index of maize, potato, and sunflower fields from unmanned aerial vehicle hyperspectral data. International Journal of Applied Earth Observation and Geoinformation 26(0), pp. $12-20$.

Feret, J.-B., François, C., Asner, G. P., Gitelson, A. A., Martin, R. E., Bidel, L. P., Ustin, S. L., le Maire, G. and Jacquemoud, S., 2008. PROSPECT-4 and 5: Advances in the leaf optical properties model separating photosynthetic pigments. Remote Sensing of Environment 112(6), pp. 3030-3043.

Gerber, F., Marion, R., Olioso, A., Jacquemoud, S., da Luz, B. R. and Fabre, S., 2011. Modeling directional-hemispherical reflectance and transmittance of fresh and dry leaves from 0.4 $\mu \mathrm{m}$ to $5.7 \mu \mathrm{m}$ with the prospect-visir model. Remote Sensing of Environment 115(2), pp. 404-414.

Gitelson, A. A., Vina, A., Ciganda, V., Rundquist, D. C. and Arkebauer, T. J., 2005. Remote estimation of canopy chlorophyll content in crops. Geophysical Research Letters.

Jacquemoud, S. and Baret, F., 1990. PROSPECT: A model of leaf optical properties spectra. Remote sensing of environment 34(2), pp. 75-91.

Jacquemoud, S., Bacour, C., Poilv, H. and Frangi, J.-P., 2000. Comparison of four radiative transfer models to simulate plant canopies reflectance: Direct and inverse mode. Remote Sensing of Environment 74(3), pp. $471-481$.

Jacquemoud, S., Ustin, S., Verdebout, J., Schmuck, G., Andreoli, G. and Hosgood, B., 1996. Estimating leaf biochemistry using the PROSPECT leaf optical properties model. Remote Sensing of Environment 56(3), pp. 194 - 202.

Jacquemoud, S., Verhoef, W., Baret, F., Bacour, C., ZarcoTejada, P. J., Asner, G. P., Franois, C. and Ustin, S. L., 2009. PROSPECT+SAIL models: A review of use for vegetation characterization. Remote Sensing of Environment 113(0), pp. S56 - S66.
Jay, S., Hadoux, X., Gorretta, N. and Rabatel, G., 2014. Potential of hyperspectral imagery for nitrogen content retrieval in sugar beet leaves. In: Proc. Int. Conf. Ag. Eng., AgEng2014, Zurich, The European Society of Agricultural Engineers (EurAgEng).

Knyazikhin, Y., Schull, M. a., Stenberg, P., Mõttus, M., Rautiainen, M., Yang, Y., Marshak, A., Latorre Carmona, P., Kaufmann, R. K., Lewis, P., Disney, M. I., Vanderbilt, V., Davis, A. B., Baret, F., Jacquemoud, S., Lyapustin, A. and Myneni, R. B., 2012. Hyperspectral remote sensing of foliar nitrogen content. Proceedings of the National Academy of Sciences of the United States of America 110(3), pp. E185-92.

Latorre-Carmona, P., Knyazikhin, Y., Alonso, L., Moreno, J., Pla, F. and Yan, Y., 2014. On hyperspectral remote sensing of leaf biophysical constituents: Decoupling vegetation structure and leaf optics using CHRIS-PROBA data over crops in barrax. Geoscience and Remote Sensing Letters, IEEE 11(9), pp. $1579-1583$.

Mahlein, A.-K., Rumpf, T., Welke, P., Dehne, H.-W., Plümer, L., Steiner, U. and Oerke, E.-C., 2013. Development of spectral indices for detecting and identifying plant diseases. Remote Sensing of Environment 128, pp. 21-30.

Nicodemus, F. E., Richmond, J. C., Hsia, J. J., Ginsberg, I. W. and Limperis, T., 1977. Geometrical considerations and nomenclature for reflectance. Vol. 160, US Department of Commerce, National Bureau of Standards Washington, DC, USA.

Schaepman-Strub, G., Schaepman, M., Painter, T., Dangel, S. and Martonchik, J., 2006. Reflectance quantities in optical remote sensingdefinitions and case studies. Remote sensing of environment 103(1), pp. 27-42.

Schlemmer, M., Gitelson, A., Schepers, J., Ferguson, R., Peng, Y., Shanahan, J. and Rundquist, D., 2013. Remote estimation of nitrogen and chlorophyll contents in maize at leaf and canopy levels. International Journal of Applied Earth Observation and Geoinformation 25(0), pp. $47-54$.

Stuckens, J., Somers, B., Delalieux, S., Verstraeten, W. and Coppin, P., 2009. The impact of common assumptions on canopy radiative transfer simulations: A case study in citrus orchards. Journal of Quantitative Spectroscopy and Radiative Transfer 110(1), pp. 1-21.

Uto, K. and Kosugi, Y., 2013. Leaf parameter estimation based on leaf scale hyperspectral imagery. IEEE J. Sel. Top. Appl. Earth Obs. Remote Sens 6, pp. 699-707.

Verger, A., Vigneau, N., Chron, C., Gilliot, J.-M., Comar, A. and Baret, F., 2014. Green area index from an unmanned aerial system over wheat and rapeseed crops. Remote Sensing of Environment 152(0), pp. $654-664$.

Verhoef, W., 1984. Light scattering by leaf layers with application to canopy reflectance modeling: The SAIL model. Remote Sensing of Environment 16(2), pp. 125 - 141.

Vigneau, N., Ecarnot, M., Rabatel, G. and Roumet, P., 2011. Potential of field hyperspectral imaging as a non destructive method to assess leaf nitrogen content in wheat. Field Crops Research 122, pp. 25-31.

Zarco-Tejada, P. J., Rueda, C. and Ustin, S., 2003. Water content estimation in vegetation with modis reflectance data and model inversion methods. Remote Sensing of Environment 85(1), pp. 109-124. 